\title{
NOTAS
}

\section{PROBLEMAS DE FONOLOGIA DIALECTAL*}

0. Hace poco tiempo tuve la oportunidad de reseñar (NRFH, 22, 1973, 121-127) el libro Spanish pronunciation, theory and practice. En esa ocasión hice algunas objeciones al trabajo de J. P. Dalbor y, a la vez, consideré que sus planteamientos estaban llenos de sugerencias en cuanto al tratamiento de los fonemas del español. Añado ahora que las ideas que de allí recogí son las que me han llevado a elaborar este estudio. De acuerdo con las preguntas que me surgieron al leer Spanish pronunciation, trataré de ver, con base en siete dialectos del español actual, si es posible proponer un diasistema fonológico para los sistemas dialectales sin dejar de considerar, por una parte, las relaciones que guardan los fonemas en cada sistema y, por otra, las relaciones de estos fonemas con sus variantes fonéticas normales $\mathbf{1}$.

0.1 . En la clasificación y descripción de los fonemas consonánticos del español o de algunos de sus dialectos se han utilizado con frecuencia los rasgos fonéticos sin un criterio claro de pertinencia. Si, de acuerdo con Alarcos (p. 42; cf. también infra, § 4.1.1) las variantes de un fonema deben tener los mismos rasgos pertinentes que éste, no parece adecuado clasificar, por ejemplo, /b/, /d/, /g/ como oclusivos 2 puesto que estos fonemas tienen variantes oclusivas y fricativas -estas últimas, incluso, más frecuentes que las primeras. Lo anterior nos llevaría a pensar que las realizaciones fricativas no pertenecen a esos fonemas, o que es necesario describirlos de otra manera para incluir tales variantes.

En el caso de $/ \mathrm{b} /, / \mathrm{d} /, / \mathrm{g} /$, se están considerando implícitamente sólo las posiciones inicial absoluta y tras nasal, en las cuales ocurren normalmente las variantes oclusivas. Esta consideración podría ser válida si se aplicara para todos los fonemas. Sin embargo, resulta contra-

* Este artículo se presentó como ponencia en el V Congreso Internacional de Hispanistas (Burdeos, 2 al 8 de septiembre de 1974). Se publica en la NRFH con autorización de los organizadores del Congreso.-Agradezco a $\mathrm{K}$. Heger y a J. McMenamin las sugerencias que me hicieron a propósito de este trabajo.

1 Utilizo los términos sistema y norma en el sentido que les da Coseriu. Para la pronunciación normal usaré los términos variante o realización fonéticas.

2 Asi lo hacen Dalbor, Canfield, Flórez, Battini (1949), Matluck y Quilis y Fernández, por sólo citar algunos. 
dictoria ya que el fonema / $/$ / debería ser descrito como africado $u$ oclusivo y algunos autores lo consideran fricativo ${ }^{3}$.

También de manera implícita se toma en cuenta sólo una posición combinatoria cuando se caracterizan como alveolares los fonemas /1/ $\mathrm{y} / \mathrm{n} / \mathrm{y}$ como sordo /s/ puesto que para / 1 / se consideran realizaciones interdentales y palatales; para / $\mathbf{n} /$ articulaciones que van de bilabiales a velares; y para / $/$ / variantes sordas y sonoras.

0.2. Los puntos de articulación tampoco han sido utilizados con criterio de pertinencia. Tanto Canfield ${ }^{4}$ como Dalbor incluyen los órdenes labial (o bilabial), labiodental, interdental, dental, alveolar, palatal, velar y faríngeo (o glotal) -en Dalbor, además, aparece un orden bilabiovelar. Estos órdenes serían pertinentes sólo si hubiera fonemas de la misma clase en cada uno de ellos. Es decir, sería necesario que hubiera en español, pongamos por caso, fonemas oclusivos sordos en cada uno de los puntos de articulación, y no los hay. Para las clases de fonemas que proponen Dalbor y Canfield bastarian los órdenes labial, dentoalveolar, palatal y velar5.

0.3. Por otra parte, aun sin considerar las objeciones anteriores, el cuadro de fonemas consonánticos que presenta Dalbor (p. 276) como general del español sería válido sólo para el dialecto de Burgos, ya que en los sistemas donde no existe el fonema palatal lateral -que, por limitaciones tipográficas, transcribiré $/ \lambda /-$, el fonema $/ 1 /$ resultaría hipercaracterizado si se describiera como alveolar, puesto que no se opone a otro fonema lateral no alveolar. De la misma forma, en los sistemas donde no existe la oposición $/ \theta /: / \mathrm{s} /$, el fonema único $/ s /$ no tendría por qué describirse como alveolar, puesto que no se opone a un fonema dental de la misma clase, sino como dentoalveolar $-y$, de hecho, las variantes de este fonema van desde interdentales hasta alveolares. Para decirlo en términos de WEINREICH, al construir sistemas generales o "diasistemas" se ignoran "las estructuras de las variedades constituyentes. En otras palabras, la dialectología actual generalmente compara elementos pertenecientes a diferentes sistemas sin destacar suficientemente su íntima ubicación en esos sistemas" (p. 309).

1. "Un «diasistema»-dice WeINREICH, p. 307-puede ser construido por el lingüista a partir de dos ["o más", p. 314] sistemas cualesquiera que tengan similaridades parciales". Para mostrar su concepción de diasistema Weinreich usa, entre otros, el ejemplo de dos dialectos que tie-

3 Así lo hacen Dalbor, Canfield y Quilis y Fernández. Flórez organiza los fonemas por modos (clases) y puntos de articulación (ordenes), mezclando unos y otros, pues habla de fonemas oclusivos (sonoros y sordos), fricativos (sordos), laterales y vibrantes, palatales y nasales. El hecho de que $/ \lambda /$ sea palatal (lateral), / $/$ / fricativo u oclusivo y / $\tilde{n} /$ palatal muestra la ambigüedad de la clasificación. Algo semejante hacen Matluck y Battini (1949).

4 Es necesario señalar que CANFIeld (p. 55) no propone fonemas para todos los órdenes. Algunos de ellos los utiliza sólo para clasificar variantes fonéticas.

5 Así lo propuso J. P. Rona en un curso que dictó en 1965 en El Colegio de México. Algo semejante hace Perissinotto para los fonemas del español de la ciudad de México. Sin embargo, como este autor no intenta una clasificación general de los fonemas - los va describiendo uno a uno-, considera órdenes diferentes el dental y el alveolar. 
nen, cada uno, un sistema de cinco vocales, pero en el primero la segunda vocal es /e/ y en el otro / $\varepsilon /$ (p. 312).

$$
1,2 / / i-\frac{x e}{2 \varepsilon}-a-o-u / /
$$

Es decir, los fonemas /e/ y $/ \varepsilon /$ se encuentran entre sí en oposición sintomática, mientras que, dentro de su sistema, cada uno de ellos está en oposición simbólica con los demás. En el diasistema ambas vocales constituyen una sola unidad. A esas unidades del diasistema las llamaré elementos, ya correspondan en los sistemas a dos fonemas o a uno solo. A estos elementos del diasistema los pondré entre barras dobles para diferenciarlos de los fonemas que, como se acostumbra, colocaré entre barras simples. Así pues, retranscribo el ejemplo de Weinreich de la siguiente manera:

$$
\begin{aligned}
& / / \mathrm{i} / / / \mathrm{e} / / / / \mathrm{a} / / / / \mathrm{o} / / / \mathrm{u} / / \\
& \text { donde } / / \mathrm{e} / / \rightarrow \begin{cases}/ \mathrm{e} / & / \mathrm{S}_{1} \\
/ \varepsilon / & / \mathrm{S}_{2}\end{cases}
\end{aligned}
$$

y todos los demás elementos corresponden unívocamente a un mismo fonema en ambos sistemas.

1.1. Por otra parte, en el caso de dos dialectos en los cuales a los fonemas /a:/ : / a/del primero corresponde el fonema /a/del segundo, utilizaré como elementos del diasistema los correspondientes al número máximo de fonemas en oposición simbólica. Por lo tanto, consideraré como elementos //a:// y //a//, de manera que el ejemplo de WEINREIcH (p. 312) -que doy a continuación simplificado-

$$
1,2 / \mid \frac{1 / a:-a /}{3 / a /} / /
$$

lo retranscribo como

donde

$$
/ / \mathrm{a}: / / / \mathrm{a} / /
$$

y

$$
/ / a: / / \rightarrow \begin{cases}/ a: / & / S_{1} \\ / a / 1 & / S_{2}\end{cases}
$$

$$
/ / a / / \rightarrow \begin{cases}/ a / 2 & / S_{1} \\ / a / 1 & / S_{1}\end{cases}
$$

e interpreto que $/ \mathbf{a} / \mathrm{s}$ es diferente $\mathrm{a} / \mathrm{a} / \mathrm{z}$, pues en el caso de que $/ \mathbf{a} / \mathbf{2}$ se oponga bilateralmente a $/ \mathrm{a} / \mathrm{s}$, el fonema $/ \mathrm{a} / \mathrm{s}$ correspondiente en el sistema 2 a los dos fonemas del sistema 1 no necesita ser caracterizado con el rasgo [-largo], como /a/2. 
2. Para proponer el inventario de diafonemas del español me basaré en los dialectos de las siguientes localidades que he seleccionado con el fin de tener una muestra muy diversificada y que menciono en orden alfabético: Buenos Aires, Burgos, Caguas (Puerto Rico), Madrid, México (ciudad), Quito, y San Luis (Argentina) .

2.1. Para decidir cuáles son los fonemas de cada uno de los sistemas que tomaré en consideración y, a la vez, para describirlos de manera que sus rasgos distintivos no se contrapongan con los que podrían encontrarse en sus variantes, consideraré explícitamente sólo una posición combinatoria: la explosiva. Esta posición, de acuerdo con la idea de Akmmanova ( $\$$ 1.1.4.1) es la que puede considerarse la posición fuerte en español, pues en ella se logra "con la máxima claridad la mayor diferenciación de fonemas" 6 .

En efecto, en cuanto a los fonemas no vocálicos, basta la secuencia ka-a para diferenciar doce de ellos -cama, cata, casa, caza. etc.-; /1/ y $/ \lambda /$ se oponen en haya/halla; $/ \mathrm{r} /: \mid \overline{\mathrm{r}} /$ en pero/perro; $/ \mathrm{f} /: / \mathrm{p} /$ en foco/poco; y /š/ : /s/ en Xola (nombre de una calle de la ciudad de México) / sola

2.2. En el diasistema que utilizaré para los siete dialectos incluiré de acuerdo con lo anterior y con lo señalado en los $\$ \S 1$. y 1.1, un total de veinte elementos para los fonemas no vocálicos. Por otra parte, concentraré los cinco fonemas vocálicos en un solo elemento $/ / \mathrm{V} / /$. Sin embargo, para ver las relaciones que guardan los fonemas en los sistemas es necesario considerarlos y describirlos -como propone Weinreich-dentro de cada sistema. El diasistema, por lo tanto, me servirá únicamente como punto de referencia para ver a qué elemento corresponden uno $\mathrm{u}$ otro fonema. Así pues, podría identificar los veintiún elementos con números del 1 al 21, pero, por razones de claridad, utilizaré para ellos los símbolos que normalmente se emplean para los fonemas. Los elementos son:

1. $/ / \theta / /, 2 . / / \mathrm{s} / /, 3 . / / \lambda / /$, 4. $/ / \mathrm{y} / /, 5 . / / 1 / /, 6 . / / \overline{\mathrm{r}} / /$,

7. $/ / \mathrm{d} / /, 8 . / / \mathrm{r} / /, 9 . / / \mathrm{x} / /, 10 . / / \mathrm{V} / /, 11 . / / \mathrm{k} / /, 12 . / / \mathrm{s} / /$,

13. $/ / \hat{\mathrm{c}} / /, 14 . / / \mathrm{b} / /, 15 . / / \mathrm{g} / / 16 . / / \mathrm{p} / /, 17 . / / \mathrm{t} / /, 18 . / / \mathrm{f} / /$,

19. $/ / \mathrm{m} / /, 20 . / / \mathbf{n} / /, 21 . / / \tilde{\mathbf{n}} / /$.

A continuación intentaré ver, con base en los primeros trece elementos, cuál es la relación de éstos con los fonemas que les corresponden y qué diferencias se pueden establecer en los sistemas dialectales.

3. Los elementos $/ / \theta / / \mathrm{y} / / \mathrm{s} / /$ corresponden a $\operatorname{los}$ fonemas $/ \theta / \mathrm{y}$ /s/ en los sistemas en que se diferencian los signos casa/caza como sucede en Burgos $\left(S_{1}\right)$ y Madrid $\left(S_{2}\right)$. En los sistemas en que no existe la oposición $/ \theta /: / \mathrm{s} /$, el representante de ambos elementos es un solo fonema. Para diferenciar los dos casos, y dado que el fonema que equivale a la ausencia de oposición tendría un rasgo menos, llamaré a éste

6 "The strong position [...] assure with utmost clarity the greatest phoneme differentiation $[\ldots]$ the weak positions [...] are characterized by a very much reduced number of phonological relevant sound-distinctions" (ibid.).

7 Para el fonema $/ 5 /$ en México y Quito, cf. infra $\$ 7$. 
$/ \mathrm{s} / 1$ y utilizaré $/ \mathrm{s} / 2$ para el fonema opuesto a $/ \theta /$. Por lo tanto, los fonemas correspondientes a los elementos $/ / \theta / / \mathrm{y} / / \mathrm{s} / /$ son
1. $/ / \theta / / \rightarrow\left\{\begin{array}{l}/ \theta / \\ / s / 1\end{array}\right.$
2. $/ / \mathrm{s} / / \rightarrow\left\{\begin{array}{l}/ \mathrm{s} / \mathrm{s} \\ / \mathrm{s} / \mathrm{s}\end{array}\right.$

4. Los elementos $/ / \lambda / /$ y $/ / y / /$ corresponden a la oposición fonológica que se muestra en halla/haya. Estos dos signos se diferencian en Burgos $\left(\mathrm{S}_{1}\right)$ y Quito $\left(\mathrm{S}_{\mathrm{s}}\right)$, mientras que en los demás sistemas se confunden en /aya/. En este caso, el fonema correspondiente a ambos elementos es $/ y /$.

4.1. Sin embargo, en Quito los signos anteriores se pronuncian [aža] : [aya], por lo que al elemento $/ / \lambda / /$ le corresponde una realización $[\check{z}]^{8}$. Surge de aquí el problema de decidir si la variante [ž] puede atribuirse al fonema $/ \lambda /$ o si, en cambio, es necesario proponer un fonema $/ \check{z} /$.

4.1.1. Si se estudiara el sistema de Quito en sí mismo y se observara que los sonidos $[\check{z}]$ : $[y]$ tienen valor fonológico, se tendrian que proponer dos fonemas $\mid \check{z} /:$ : $/$ cuya oposición bilateral tendría como rasgos distintivos, respectivamente [+ estridente] : [- estridente $]^{9}$.

Por otra parte, como señala Alarcos (p. 42), "cada sonido presenta $[\ldots]$ los rasgos pertinentes del fonema del que es realización, más otra serie de rasgos fónicos irrelevantes, que dependen de diversas causas. Por ello un mismo fonema puede ser realizado por diversos sonidos, con tal que éstos contengan los mismos rasgos diferenciales". De acuerdo con la descripción que da el mismo autor (p. 179), el fonema $/ \lambda /$ contiene, entre otros, los rasgos $[+$ vocal, + consonante]. La variante [ž], en cambio, sería [- vocal, + consonante] $]^{10}$. De acuerdo con esto, [ž] no puede ser considerado variante de $/ \lambda /$, por lo que resulta más ade-

8 Cf. Toscano, pp. 99 y 100; Canfield, mapa V, y Boyd-Bowman, p. 224: "El resto de la sierra (con Quito, la capital) opone a la $y$ no la $\lambda$, sino una ̌̌". Yo mismo he tenido esa impresión al escuchar en Quito a algunas personas. El fenómeno ha sido registrado para Quito también por RoNA, y para Santiago del Estero por BATTINI (1964). Sin embargo, un colega me ha comunicado sus dudas sobre este hecho, ya que, históricamente, la evolución ha sido $[\lambda]>[y]>[\check{z}]$, por lo que, en un cierto momento, se habría producido la confusión con $/ y /$. Convendría, pues, hacer un estudio más a fondo. ¿Se trata, acaso, de influencia del quechua, como parece sugerir Boyd-Bowman?

9 De aquí en adelante, aunque me referiré ocasionalmente a los rasgos fonéticos de otros investigadores, utilizaré, para mis propias descripciones, los que proponen Chomsky y Halle. Después de comparar estos rasgos con los de otros autores (Jakobson, Navarro, Harms) llegué a la conclusión de que eran los que me permitían con más facilidad, adecuación y economía, describir los sistemas fonológicos de que me ocupo. Para la relación entre estos rasgos con los de Jakobson, cf. Chomsky y Halle (\$ 4.2.1). Harris (pp. 10-13) ofrece una evaluación comparativa de los rasgos de Jakobson y de Chomsky y Halle,

I0 Asi lo propone Harris (p. 42). 
cuado proponer para Quito un fonema $/ \check{z} /$ correspondiente al elemento $/ / \lambda / /$.

4.2. El fonema $/ \check{z} /$ está en oposición bilateral con el fonema $/ y /$, como antes he dicho, por los rasgos [+ estridente] : [- estridente]. Sin embargo, el rasgo [- estridente] no sería pertinente para $/ y /$ en los sistemas en los cuales no se opusiera a $/ \check{z} /$. A causa de esto, es necesario proponer para el elemento $/ / \mathrm{y} / /$ un fonema $/ \mathrm{y} / \mathrm{a}$ con el rasgo [- estridente] para Quito $\left(S_{3}\right)$ y un fonema $/ y / 1$ sin ese rasgo para los demás sistemas. Así pues, los elementos $/ / \lambda / /$ y $/ / \mathrm{y} / /$ corresponden a los siguientes fonemas
3. $/ / \lambda / / \rightarrow \begin{cases}/ \lambda / & / S_{1} \\ / \check{z} / & / S_{2} \\ / y / z & \end{cases}$
4. $/ / \mathrm{y} / / \rightarrow \begin{cases}/ \mathrm{y} / \mathrm{s} & / \mathrm{S}_{\mathrm{z}} \\ / \mathrm{y} / \mathrm{s}\end{cases}$

4.3. En Burgos $\left(S_{1}\right)$ el fonema $/ \lambda /$ está en oposición bilateral con $/ 1 /$, mediante los rasgos [- coronal] : [ coronal], mientras que en los demás sistemas no existe dicha oposición. Para no hipercaracterizar el fonema / $1 /$ en tales sistemas con el rasgo [+ coronal] y, a la vez, incluir este rasgo en los sistemas donde se opone a $/ \lambda /$, se hace necesario proponer, para el elemento $/ / 1 / /$ dos fonemas: un fonema $/ 1 / 2$ con el rasgo $[+$ coronal $]$ y un fonema $/ 1 / 1$ sin ese rasgo.

$$
\text { 5. } / / 1 / / \rightarrow\left\{\begin{array}{l}
/ 1 / 2 \\
/ 1 / x
\end{array}\right.
$$

5. La oposición fonológica que diferencia los signos carro/caro y que corresponde a los elementos $/ / \overline{\mathrm{r}} / /$ y $/ / \mathrm{r} / /$ presenta, para el primero de ellos, un buen número de variantes en los dialectos. Dentro de ellas están las realizaciones sonoras alveolares, vibrantes, fricativas o asibiladas; y las de articulación uvular, vibrantes o fricativas, características de Puerto Rico (Navarro, 1966, p. 89). Si se considera que el fonema $/ \overline{\mathbf{r}} /$ tiene los rasgos [+ vocal, + consonante] (Alarcos, ibid., Harris, p. 48), cabe la posibilidad de atribuir a él todas las variantes anteriores menos la asibilada larga - para la cual, por limitaciones de la imprenta, utilizaré el signo $\dot{r}-$, ya que ésta no participa del rasgo $[+ \text { vocal }]^{11}$. A causa de esto, la realización asibilada se opone bilateralmente a $/ \check{z} /$ o a $/ \mathrm{d} /$ pero no a $/ \mathrm{r} /$. Por las mismas razones que he dado antes $(\S 4.1)$, es necesario proponer como correspondientes a $/ / \overline{\mathbf{r}} / /$ los fonemas $/ \dot{r} /$ para Quito $\left(S_{i}\right)$ y San Luis $\left(S_{i}\right)^{12}$ y $/ \overline{\mathbf{r}} /$ para los demás sistemas.

11 Así lo considera Harris (ibid.). Cf. también la opinión de Navarro (1963, $\S 117)$ : la variante asibilada se diferencia de la fricativa "por ser menos sonora y vocálica".

12 Cf. Toscano (\$52) para Quito y Bartini $(1949, \S 42)$ para San Luis. 
6. $/ / \overline{\mathrm{r}} / / \rightarrow\left\{\begin{array}{l}|\ddot{r}| \\ \mid \overline{\mathrm{r}} /\end{array} / \mathrm{S}_{3}, S_{4}\right.$

5.1. El fonema $\mid \check{r} /$ puede aparecer en oposición bilateral con $/ \mathrm{d} /$ o con $/ \check{z} /$, según se lo considere [+ anterior] o [- anterior]. Para cada caso es posible encontrar rasgos diferenciales. Así, si $/ \check{r} /$ es $[+$ anterior] se diferenciaría de $/ d /$ por el rasgo [+ estridente]. Frente a $/ \check{\mathbf{z}} /$, en cambio, sería necesario caracterizar $\mid \check{r} /$ como [- distribuido].

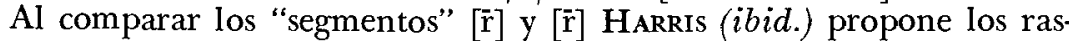
gos [- anterior] para $[\overline{\mathbf{r}}]$ y [- anterior $]$ para $[\check{r}]$. En contra de su opinión, creo que difícilmente podría haber una mayor o menor 'anterioridad' entre ambas realizaciones, e, incluso, podría pensarse que [rir] es

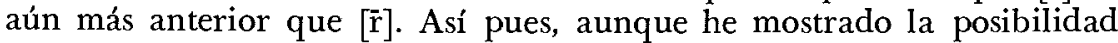
de diferenciar bilateralmente tanto $|\check{r}|: / \mathrm{d} /$ como $|\check{r}|: \mid \check{z} /{ }^{13}$, creo más adecuado considerar $/ \check{r} /$ como [- anterior].

5.1.1. Al oponerse bilateralmente a /d/ se hace necesario considerar, a la vez, que en los sistemas donde a $/ \dot{r} /$ corresponde $/ \overline{\mathbf{r}} /$, el rasgo [- estridente] en /d/ resulta redundante. Para evitarlo hace falta proponer para el elemento $/ / \mathrm{d} / /$ un fonema $/ \mathrm{d} / \mathrm{s}$ con ese rasgo y un fonema $/ d / 1$ sin él.

7. $/ / \mathrm{d} / / \rightarrow\left\{\begin{array}{l}/ \mathrm{d} / \mathrm{s} \\ / \mathrm{d} / \mathrm{s}\end{array}\right.$

5.1.2. En forma similar, el rasgo [- tenso] sólo es necesario en $/ \mathrm{r} /$ para diferenciarlo bilateralmente de $/ \overline{\mathbf{r}} /$. En los sistemas con $/ \check{r} /$, en cambio, ese rasgo no es pertinente. Para poder tomar en cuenta lo anterior, es suficiente considerar, para el elemento $/ / \mathrm{r} / /$, los fonemas $/ \mathrm{r} / \mathrm{s}$ con el rasgo [- tenso] y $/ r /$, sin dicho rasgo.

8. $/ / \mathrm{r} / / \rightarrow\left\{\begin{array}{l}/ \mathrm{r} / \mathrm{1} \\ / \mathrm{r} / \mathrm{z}\end{array}\right.$

6. Para el elemento $/ / \mathrm{x} / /$ existen, además de las intermedias, las realizaciones velar $[\mathrm{x}]$ y faríngea $[\mathrm{h}]$-esta última, por ejemplo, se ha registrado en Caguas $\left(S_{5}\right)$. Entre una y otra de las variantes hay una diferencia importante: ambas comparten el rasgo [-vocal], pero la segunda es [- consonante] (Chomsky y Halle, § 3.3). Debido a esto, y de acuerdo con lo que he dicho supra (\$\$ 4.1 y 5 ) al elemento $/ / \mathbf{x} / /$ le corresponden los fonemas $/ \mathrm{x} / \mathrm{y} / \mathrm{h} /{ }^{14}$.

9. $/ / \mathrm{x} / / \rightarrow\left\{\begin{array}{l}/ \mathrm{h} / \\ / \mathrm{x} /\end{array}\right.$

13 Oposición que, por cierto, Harris no distingue.

14 Con esto le doy la razón a Dalbor, quien propuso también un fonema /h/ para algunos dialectos del español. 
6.1. El fonema /h/ puede ser descrito como [+ sonorante, - nasal, - consonante], por lo que se diferenciaría de los fonemas vocálicos - para los cuales, como he dicho, utilizaré el signo $/ \mathrm{V} /-$ mediante el rasgo [- vocal].

6.1.1. Al lado de esto, para evitar la hipercaracterización de /V/ en los sistemas donde a $/ \mathrm{h} /$ corresponde $/ \mathrm{x} /$, es suficiente proponer $/ \mathrm{V} / \mathrm{s}$ sin el rasgo [+ vocal] y $/ \mathrm{V} / 2$ con ese rasgo para el elemento $/ / \mathrm{V} / /$.

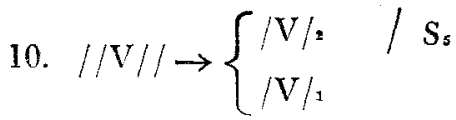

6.2. Por otra parte, como $/ \mathrm{x} /$ está en oposición bilateral con $/ \mathrm{k} /$ mediante los rasgos [+ continuo] : [- continuo], en los sistemas donde a $/ x /$ corresponde $/ h /$, el rasgo $[-$ continuo $]$ es redundante en $/ k /$. Como en casos anteriores, es necesario proponer, para el elemento $/ / \mathrm{k} / /$, un fonema $/ \mathrm{k} / \mathrm{2}$ caracterizado por el rasgo [- continuo] y un fonema $/ \mathrm{k} / \mathrm{s}$ sin ese rasgo.

11. $/ / \mathrm{k} / / \rightarrow\left\{\begin{array}{l}/ \mathrm{k} / \mathrm{s} / \mathrm{S}_{\text {。 }} \\ / \mathrm{k} / \mathrm{2}\end{array}\right.$

7. Se puede considerar que existe un fonema $/ \check{s} /$, en las ciudades de Quito ${ }^{15}$ y México $^{16}$, por lo general en voces indígenas integradas al léxico español. El fonema es muy poco frecuente y, por lo mismo, es difícil encontrar parejas mínimas que muestren su valor diferencial. No obstante, para Quito $\left(S_{3}\right)$ existe la pareja / kaši/ (hipocorístico de Casimiro): / kási /; y, para México ( $\left.\mathrm{S}_{6}\right)$ además de la ya citada $(\$ 2.1)$, /ĉicici/ ('pecho, ubre'): / šiši/ ('especie de jabón vegetal') ${ }^{17}$. Con base en esto -y así sea provisionalmente- decidí incluir en el diasistema el elemento $/ / \check{s} / /$.

15 Toscano (\$ $\$ 4$ ) registra como general en Ecuador shigra 'bolsa' y su derivado shigrero, que tiene gramemas españoles. En la Sierra -dice Toscano- "existen muchas palabras con sh: pishcochaqui ('pie de pájaro', literalmente $=$ un artificio de riego) $[\ldots]$ en apellidos y toponímicos [...] la $s$ de algunas palabras castellanas suele cambiarse en sh: en hipocorísticos como Pashi (Pacífico), Pishi (Purificación), Shuli (Soledad), Cashi (Casimiro), etc., sobre todo en el habla de los indios. Asimismo se dice ordinariamente dieshocho o dishocho (dieciocho)". Cf. también Boyd-Bowman, pp. 228 y 233.

16 Cf. Lope Blanch (p. 95) y Perissinotto (p. 64). Entre las voces que citan estos autores se encuentran, además de Xola y xixi, Mixcoac, Mexica y mixiote. Perissinotto añade que en el sonido [s] aparece también en algunas pocas palabras extranjeras de uso muy frecuente, como garage [garaš], beige [beiš], show [šow] y chic [šik], lo que muestra, por lo menos, que los mexicanos no tienen ninguna dificultad en pronunciarlo. En cuanto a las voces indígenas, obsérvese que no todas son topónimos o nombres propios. Mexica se utiliza para referirse a un "individuo de la tribu primitiva de los mexicas" y mixiote es la "epidermis de la penca del maguey" (SANTAMARia, ss. vv. mexica, mixiote). El mixiote se aprovecha para envolver carne de pollo o de puerco. El atado después se cuece. De allf que en la ciudad de México se haya popularizado el término mixiote para designar ese tipo de comida. Algunas de las voces indigenas, además, pueden tomar gramemas españoles. Se dice, por ejemplo, "Compra dos mixiotes" y "Los mexicas vivian en el Valle de México":

17 LOPE Blanch, ibid. 
7.1. Sin embargo, el elemento //š// no es común a todos los sistemas, ya que los signos que he utilizado como ejemplos no se han registrado fuera de los lugares que menciono. En los demás sistemas, por lo tanto, dicho elemento no existe ${ }^{18}$. La relación sería la siguiente:

$$
\text { 12. } / / \check{s} / / \rightarrow\left\{\begin{array}{l}
/ \check{s} / / S_{s}, S_{s} \\
\text { no existe }
\end{array}\right.
$$

7.2. En los sistemas para los cuales he propuesto el fonema /š/, éste se opone a $/ \hat{c} /$ mediante los rasgos $[+$ continuo $]:[-$ continuo]. Dado que la caracterización de $/ \hat{c} /$ como $[-$ continuo $]$ es innecesaria en los sistemas donde $/ \check{\mathrm{s}} /$ no existe, al elemento $/ / \hat{\mathrm{c}} / /$ le correspondería un fonema $/ \hat{c} / 2$, con el rasgo mencionado para $S_{s}$ y $S_{a}$ y un fonema $/ \hat{c} / s \sin$ sin ese rasgo para los demás sistemas.

13. $/ / \hat{\mathrm{c}} / / \rightarrow\left\{\begin{array}{l}/ \hat{\mathbf{c}} / \mathrm{z} \\ / \hat{\mathrm{c}} / \mathrm{x}\end{array}\right.$

8. Los demás elementos del diasistema a los cuales no me he referido los consideraré en relación biunívoca con los fonemas de cada uno de los siete sistemas que he tomado en cuenta en este trabajo. Las correspondencias, por lo tanto son
14. $/ / \mathrm{b} / / \rightarrow / \mathrm{b} /$
18. $/ / \mathrm{f} / / \rightarrow / \mathrm{f} /$
15. $/ / \mathrm{g} / / \rightarrow / \mathrm{g} /$
19. $/ / \mathrm{m} / / \rightarrow / \mathrm{m} /$
16. $/ / \mathrm{p} / / \rightarrow / \mathrm{p} /$
20. $/ / \mathrm{n} / / \rightarrow / \mathrm{n} /$
17. $/ / \mathrm{t} / / \rightarrow / \mathrm{t} /$
21. $/ / \tilde{\mathbf{n}} / / \rightarrow / \tilde{\mathbf{n}} /$

9. Las tablas 1,2 y 3 , que aparecen más adelante, obran a manera de conclusión. En la tabla 1 aparecen los trece primeros elementos del diasistema y los fonemas que les corresponden en cada uno de los sistemas. En la tabla se leen las oposiciones simbólicas en forma vertical y las sintomáticas en forma horizontal.

La tabla 2 es una recapitulación de las reglas de correspondencia entre los elementos del diasistema y los fonemas que he propuesto para cada sistema dialectal.

En la tabla 3, por último, aparece la descripción de todos los fonemas que he mencionado para los veintiún elementos del diasistema ${ }^{19}$. He tratado de hacer las descripciones de los fonemas de la manera más económica que me fue posible encontrar y en forma tal que sus rasgos distintivos correspondan también a los de las variantes que aparecen en posición explosiva. Con base en esto, los dialectos que tengan el mis-

18 Naturalmente, cabe la posibilidad de que exista en algún dialecto. Sin embargo, no lo he encontrado para otros lugares en la bibliografía consultada.

19 Como antes he dicho (cf. supra, nota 9), utilizo los rasgos de Chomsky y Halle, que traduzco al español. El único rasgo que me ofreció problemas en la traducción fue back, para el cual usé retraido. Por otra parte, creo que el rasgo [+ lateral] es aplicable, al menos en español, también para el fonema palatal $/ \lambda /$ y no sólo para el alveolar $/ 1 /$, como proponen Chomsky y Halle. 
mo sistema podrán diferenciarse mediante la oposición sintomática de las variantes. Para ello podrían utilizarse, por ejemplo, los fonemas $/ \mathrm{s} / \mathrm{x}$, $/ \mathrm{y} / \mathrm{1}, / \hat{\mathrm{c}} / \mathbf{1}$ y $/ \overline{\mathrm{r}} /$, ya que $/ \mathrm{s} / \mathrm{s}$ incluye realizaciones que van desde interdentales hasta alveolares; $/ \mathrm{y} / \mathrm{s}$ variantes tanto estridentes como no estridentes; $/ \hat{\mathbf{c}} / \mathbf{2}$ sonidos continuos y no continuos; $\mathrm{y} / \overline{\mathrm{r}} /$ variantes sonoras alveolares y uvulares, fricativas o vibrantes.

El Colegio de México.

Raúl Ávila

TABla 1. Oposiciones SIMbólicas y sintomáticas

\begin{tabular}{|c|c|c|c|c|c|c|c|}
\hline & $S_{\mathfrak{x}}$ & $S_{z}$ & $S_{2}$ & S. & $S_{s}$ & $S_{6}$ & $S_{7}$ \\
\hline 1. $/ / \theta / /$ & \multicolumn{2}{|c|}{$|\theta|$} & & & \multirow{2}{*}{\multicolumn{3}{|c|}{$/ \mathrm{s} / \mathrm{s}$}} \\
\hline 2. $/ / \mathrm{s} / /$ & \multicolumn{2}{|c|}{$/ \mathrm{s} / \mathrm{s}$} & & & & & \\
\hline 3. $/ / \lambda / \mid$ & $|\lambda|$ & \multirow{2}{*}{$/ y / 1$} & $|\check{z}|$ & & \multirow{2}{*}{\multicolumn{2}{|c|}{$/ y / s$}} & \\
\hline 4. $/ / \mathrm{y} / /$ & $/ y / 2$ & & $/ y / s$ & & & & \\
\hline 5. $/ / 1 / /$ & $/ 1 / 2$ & \multicolumn{6}{|c|}{$/ 1 / 1$} \\
\hline 6. $/ / \overline{\mathbf{r}} / /$ & \multicolumn{2}{|c|}{$|\overline{\mathbf{r}}|$} & \multicolumn{2}{|c|}{$|\check{r}|$} & \multicolumn{3}{|c|}{$\mid \overline{\mathbf{r}} /$} \\
\hline 7. $/ / \mathrm{d} / /$ & \multicolumn{2}{|c|}{$/ \mathrm{d} / \mathrm{s}$} & \multicolumn{2}{|c|}{$/ \mathrm{d} / \mathrm{s}$} & \multicolumn{3}{|c|}{$/ \mathbf{d} / 1$} \\
\hline 8. $/ / \mathrm{r} / /$ & \multicolumn{2}{|c|}{$/ \mathrm{r} / \mathrm{s}$} & \multicolumn{2}{|c|}{$/ \mathbf{r} / \mathbf{2}$} & \multicolumn{3}{|c|}{$/ \mathrm{r} / \mathrm{s}$} \\
\hline 9. $/ / x / /$ & \multicolumn{4}{|c|}{$|x|$} & $/ \mathrm{h} /$ & \multicolumn{2}{|c|}{$|\mathbf{x}|$} \\
\hline $10 . / / \mathrm{V} / /$ & \multicolumn{4}{|c|}{$/ \mathrm{V} / \mathrm{s}$} & $/ \mathrm{V} / \mathrm{z}$ & \multicolumn{2}{|c|}{$/ \mathrm{V} / \mathrm{s}$} \\
\hline 11. $/ / \mathrm{k} / /$ & \multicolumn{4}{|c|}{$/ \mathrm{k} / \mathrm{s}$} & $/ \mathrm{k} / \mathrm{s}$ & \multicolumn{2}{|c|}{$/ \mathrm{k} / \mathrm{s}$} \\
\hline 12. $/ / \check{s} / /$ & \multicolumn{2}{|c|}{ no existe } & $|\check{s}|$ & \multicolumn{2}{|c|}{ no existe } & $\mid \check{s} /$ & n. e. \\
\hline 13. $/ / \hat{\mathbf{c}} / /$ & \multicolumn{2}{|c|}{$/ \hat{\mathbf{C}} / \mathbf{2}$} & $\mid \hat{c} / 2$ & \multicolumn{2}{|c|}{$/ \hat{\mathrm{c}} / \mathbf{1}$} & $/ \hat{c} / 2$ & $/ \hat{\mathrm{C}} / \mathrm{r}$ \\
\hline
\end{tabular}

$\mathrm{S}_{1}$ : Burgos; $\mathrm{S}_{2}$ : Madrid; $\mathrm{S}_{2}:$ Quito; $\mathrm{S}_{4}$ : San Luis (Argentina); $\mathrm{S}^{6}$ : Caguas; $\mathrm{S}_{\mathbf{8}}$ : México (ciudad); $S_{q}$ : Buenos Aires. 
Tabla 2. Recapttulación de las reglas de correspondencia ENTRE ELEMENTOS Y FONEMAS

1. $/ / \theta / / \rightarrow\left\{\begin{array}{l}/ \theta / \\ / s / \mathrm{s}\end{array}\right.$

2. $/ / \mathrm{s} / / \rightarrow\left\{\begin{array}{l}/ \mathrm{s} / 2 \\ / \mathrm{s} / \mathrm{s}\end{array}\right.$

3. $/ / \lambda / / \rightarrow \begin{cases}/ \lambda / & / S_{1} \\ / \check{z} / & / S_{3} \\ / y / 1 & \end{cases}$

4. $/ / \mathrm{y} / / \rightarrow\left\{\begin{array}{l}/ \mathrm{y} / \mathrm{s} / \mathrm{S} s \\ / \mathrm{y} / \mathrm{s}\end{array}\right.$

5. $/ / 1 / / \rightarrow\left\{\begin{array}{l}/ 1 / \mathrm{s} / \mathrm{S}_{x} \\ / 1 / \mathrm{s}\end{array}\right.$

6. $/ / \overline{\mathrm{r}} / / \rightarrow\left\{\begin{array}{l}\mid \check{r} / \\ / \overline{\mathrm{r}} /\end{array} / \mathrm{S}_{s}, \mathrm{~S}_{\mathbf{s}}\right.$

7. $/ / \mathrm{d} / / \rightarrow\left\{\begin{array}{l}/ \mathrm{d} / \mathrm{s} / \mathrm{S}, \mathrm{s}, \\ / \mathrm{d} / \mathrm{s}\end{array}\right.$

8. $/ / \mathrm{r} / / \rightarrow\left\{\begin{array}{l}/ \mathrm{r} / \mathrm{s} \\ / \mathrm{r} / \mathrm{s}\end{array}\right.$

9. $/ / \mathrm{x} / / \rightarrow\left\{\begin{array}{l}/ \mathrm{h} / \\ / \mathrm{x} / \mathrm{S}\end{array}\right.$
10. $/ / \mathrm{V} / / \rightarrow\left\{\begin{array}{l}/ \mathrm{V} / 2 / \mathrm{S}_{\mathrm{s}} \\ / \mathrm{V} / \mathrm{s}\end{array}\right.$

11. $/ / \mathbf{k} / / \rightarrow\left\{\begin{array}{l}/ \mathrm{k} / \mathrm{s} / \mathrm{S}_{5} \\ / \mathrm{k} / \mathrm{a}\end{array}\right.$

12. $/ / \check{s} / / \rightarrow\left\{\begin{array}{l}/ \check{s} / / S_{s}, S_{\triangleleft} \\ \text { no existe }\end{array}\right.$

13. $/ / \hat{\mathrm{c}} / / \rightarrow\left\{\begin{array}{l}/ \hat{\mathrm{c}} / 2 \\ / \hat{\mathrm{c}} / \mathrm{s}\end{array}\right.$

14. $/ / \mathrm{b} / / \rightarrow\{/ \mathrm{b} /$

15. $/ / \mathrm{g} / / \rightarrow\{\mid \mathrm{g} /$

16. $/ / \mathrm{p} / / \rightarrow\{/ \mathrm{p} /$

17. $/ / \mathrm{t} / / \rightarrow\{/ \mathrm{t} /$

18. $/ / \mathrm{f} / / \rightarrow\{/ \mathrm{f} /$

19. $/ / \mathrm{m} / / \rightarrow\{/ \mathrm{m} /$

20. $/ / \mathrm{n} / / \rightarrow\{/ \mathrm{h} /$

21. $/ / \tilde{\mathbf{n}} / / \rightarrow\{/ \tilde{\mathbf{n}} /$ 


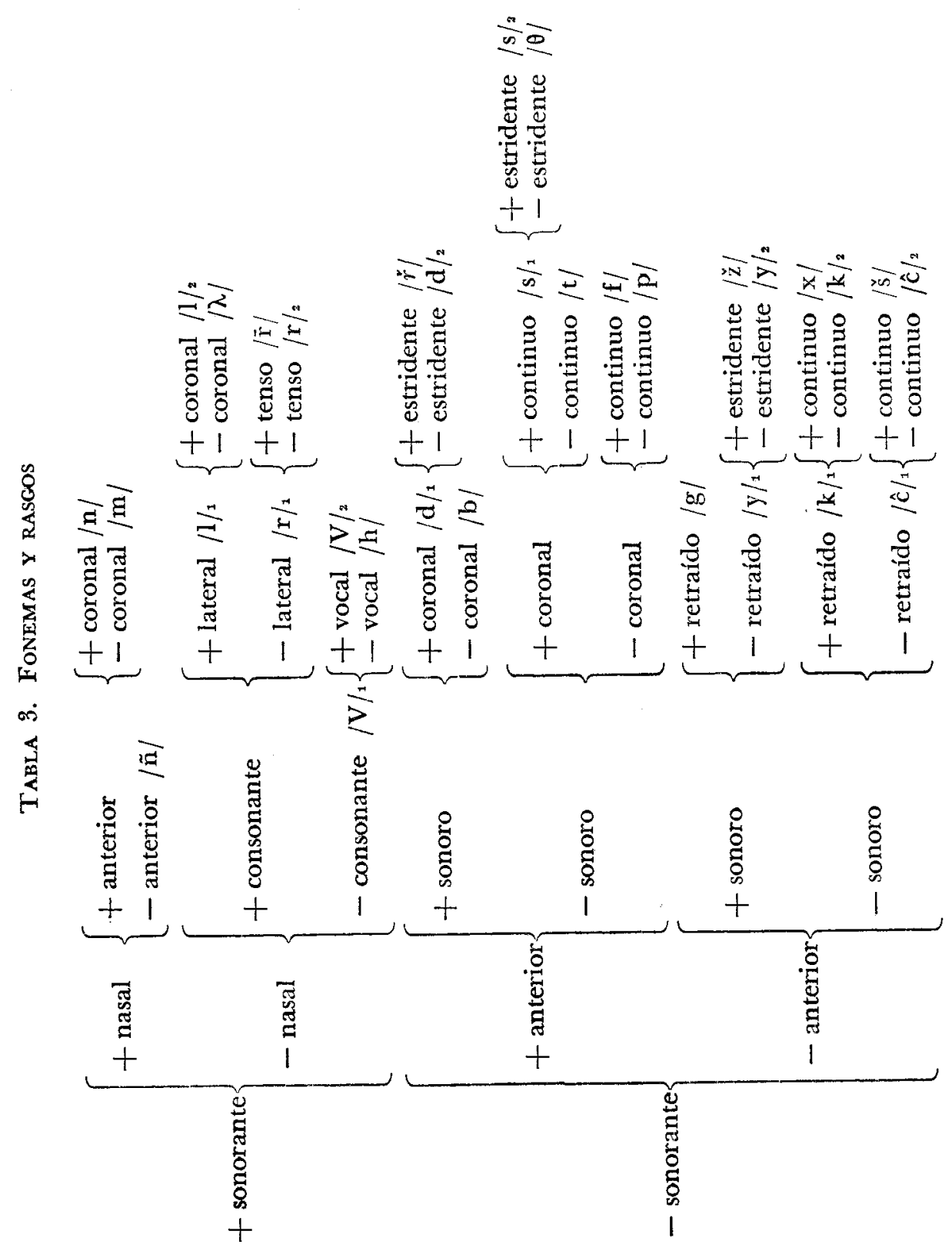




\section{ABREVIATURAS DE OBRAS CITADAS}

Akhmanova $=$ Olga Akhmanova, Phonology, morphonology, morphology, The Hague, 1971.

Alarcos = Emilio Alarcos Llorach, Fonologia española, 4a ed., Madrid, 1965.

Battin $(1949)=$ B. E. Vidal de BatTinI, El habla rural de San Luis, t. 1, Buenos Aires, 1949

$(1964)=$ B. E. Vrdal de Batrini, "El español de la Argentina", Presente $y$ futuro de la lengua española, t. 1, Madrid, 1964, 183-192.

Boyd-Bowman $=$ P. Boyd-Bowman, "Sobre la pronunciación del español en el Ecuador", NRFH, 7 (1953), 221-233.

Coserru = E. Coserru, "Sistema, norma y habla", en su libro Teoria del lenguaje y lingüistica general (Madrid, 1962), 11-113.

Chomsky y Halle $=$ N. Chomsky y M. Halle, "The phonetic framework", en su libro The sound pattern of English, New York, 1968, cap. 7.

Dalbor $=$ John B. Dalbor, Spanish pronunciation. Theory and practice, New York, 1969.

Flórez = Luis Flórez, La pronunciación del español en Bogotá, Bogotá, 1951.

HARMS $=$ Robert T. HARMs, Introduction to phonological theory, Englewood Cliffs, N. J., 1968.

HaRRIS = JAMES W. HaRRIs, Spanish phonology, Cambridge, Mass., 1969

Jakobson $=$ Roman Jakobson y Morris HaLle, Fundamentos del lenguaje, Madrid, 1967.

Lope Blanch $=\mathrm{J}$. M. Lope Blanch, "La influencia del sustrato en la fonética del español de México", en su libro Estudios sobre el español de México, México, 1972, 93-107.

Matluck = J. H. Matluck, "La pronunciación del español en el Valle de México", NRFH, 6 (1952), 109-120.

Navarro (1963) = Tomás Navarro, Manual de pronunciación española, 1la ed., Madrid, 1963.

(1966) = Tomás Navarro, El español en Puerto Rico, 2a ed., Rio Piedras, 1966.

Perissinottó $=$ Grorgio Perissinotto, The phonology of the Spanish spoken in Mexico City [Tesis, Columbia University, 1971].

Quilis y Fernández = Antonio Quilis y Joseph A. Fernández, Curso de fonética y fonologia españolas, 2a ed., Madrid, 1966.

RoNA $=$ J. P. RoNA, "El problema de la división del español americano en zonas dialectales", Presente y futuro de la lengua española, t. 1, Madrid, 1964, 215-226.

Santamaría $=$ Francisco J. Santamaría, Diccionario de mejicanismos, México, 1959.

Toscano = Humberto Toscano Mateus, El español en el Ecuador, Madrid, 1953.

WeINREICH $=$ U. WeINREICH, "Is a structural dialectology possible?", en J. A. Fish. man (ed.), Readings in the sociology of language, The Hague, 1970, 305-319.

\section{LOS CONCEPTOS 'PERFECTIVO' Y 'PERFECTO' EN EL SISTEMA VERBAL DEL CASTELLANO MODERNO}

Samuel Gili y Gaya refuta ${ }^{1}$ en su Curso superior de sintaxis espanola ${ }^{2}$ la definición del pretérito indefinido que ofrece la Gramática de la Real Academia Española ${ }^{3}$ donde se lee lo siguiente: "Como tiempo

1 I herewith express my sincere appreciation to the Research Committee of the University of Delaware for the Summer Research Grant awarded to me in 1973, which enabled me to complete this research in the field of Spanish philology.

2 Barcelona, 1969, p. 149.

3 Madrid, 1959, p. 270. 